\title{
Formation probabilities in quantum critical chains and Casimir effect
}

\author{
M. A. Rajabpour \\ Instituto de Física, Universidade Federal Fluminense, Av. Gal. Milton Tavares de Souza \\ s/n, Gragoatá, 24210-346, Niterói, RJ, Brazil \\ PACS 68.35.Rh - Phase transitions and critical phenomena \\ PACS 11.25. Hf - Conformal field theory, algebraic structures \\ PACS 05.40.-a - Fluctuation phenomena, random processes, noise, and Brownian motion
}

\begin{abstract}
We find a connection between logarithmic formation probabilities of two disjoint intervals of quantum critical spin chains and the Casimir energy of two aligned needles in two dimensional classical critical systems. Using this connection we provide a formula for the logarithmic formation probability of two disjoint intervals in generic $1+1$ dimensional critical systems. The quantity is depenedent on the full structure of the underlying conformal field theory and so useful to find the universality class of the critical system. The connection that we find also provides a very efficient numerical method to calculate the Casimir energy between needles using quantum critical chains. The agreement between numerical results performed on critical transverse field Ising model and XX chain with our exact results is very good. We also comment on the mutual Rényi information of two disjoint intervals.
\end{abstract}

The subjects of quantum phase transitions and finding the universality class of the critical points have been of great interest in recent years both for theoretical reasons and also because of the recent experimental realization of many low-dimensional quantum systems. In quantum chain studies the tradition is to find the critical point, then the central charge of the underlying conformal field theory and finally critical exponents of the system. The same procedure also works for two dimensional classical systems. Since the birth of conformal field theory, different methods were invented to follow the above procedure with each of them having their own advantages and disadvantages. For example, one of the early methods to calculate the central charge was the transfer matrix technique [1] which, although very efficient in numerical calculations, would not give the central charge without having the sound velocity. Calculations based on entanglement entropy [2] does not suffer from this problem, however, they are very difficult to measure in actual experiments. The recent investigations based on Shannon mutual information [3] seem to be promising for experimental investigations but at the same time they are basis dependent quantities. Similar arguments are also valid in calculating extra structures of different universality classes such as the critical exponents. Since diverse methods are useful in different setups it seems to be natural to investigate wide range of methods for finding the universality class of the system.

Consider the ground state of a critical spin chain $\left|\psi_{G}\right\rangle=\sum_{I} a_{I}|I\rangle$ written in a particular local basis, where $I$ here stands for particular configuration. The logarithmic formation probability in this basis is defined as the logarithm of the probability of having particular configuration $p_{I}=\left|a_{I}\right|^{2}$ in the domain $D$. When $D$ is a connected domain and the corre- 
sponding basis is the $\sigma^{z}$ basis and the desired configuration is a sequence of up (down) spins, this quantity is called emptiness formation probability and has been studied in many critical and non-critical spin chains in the last couple of decades $4-10$. The results of many analytical calculations suggest that the emptiness formation probability of critical systems decays like a Gaussian (exponential) for systems with (without) $U(1)$ symmetry. The exponential is accompanied with a power-law with an exponent which is universal and as we mention later dependent on the central charge 9]. In other words by calculating the emptiness formation probability one can gain information about the universality class of the system. Although knowing the central charge of the system helps in getting some information regarding the universality class of the system, in principle, it is not enough to determine it uniquely. To fix the universality class, one needs to also have information regarding the scaling operators of the system. In this letter, we will show that extra information can be achieved by studying the formation probabilities of two disjoint intervals. From this perspective, our quantity of interest plays the same role as the entanglement entropy. The entanglement entropy of a connected subsystem fixes the central charge and the same quantity for two disjoint intervals fixes the operator content of the system, see 11] and references therein. To calculate the formation probability of two disjoint intervals, we first map the system to the problem of the Casimir free energy of two aligned needles. A great deal is known about the form of the Casimir energy of floating objects in two dimensional medium [12 14], see also 15] and references therein. Using the results of [12,13] we give a formula for the formation probability of two disjoint intervals in one dimensional critical quantum systems. We then check our finding using the numerical technique developed in 10 for the critical Ising model and the XX chain. Our mapping between the formation probabilities and the Casimir energy of the aligned needles gives a very efficient method to also calculate numerically the Casimir energy of the needles which is extremely difficult in the usual two dimensional classical setups. For recent connection between the Casimir effect and the $n=2$ Rényi entanglement entropy see 16. The techniques based on Casimir effect have also found many applications in recent studies of non-equilibrium statistical mechanics 17 .

In 9] by mapping the problem of critical quantum chain to a two dimensional classical counterpart it was shown that the emptiness formation probability of a subregion for a spin chain can be seen as the ratio of two partition functions $\frac{Z^{\text {slit }}}{Z}$, where $Z^{\text {slit }}$ is the partition function of the whole system minus a slit and $Z$ is the total partition function. The argument goes as follows: consider a quantum periodic system with the Hamiltonian $H$. The transfer matrix of the system is $T=e^{-\epsilon H}$, where $\epsilon$ is the imaginary time step. Since in the limit of infinite steps $N \rightarrow \infty$ we have $T^{N} \sim e^{-\epsilon N E_{g}}\left|\psi_{g}><\psi_{g}\right|$ one can now calculate the emptiness formation probability of the ground state using the following formula:

$$
p_{D}=\lim _{N \rightarrow \infty} \frac{<\psi\left|T^{\frac{N}{2}} \delta\left(|\sigma>-| \uparrow \ldots \uparrow>_{b}\right) T^{\frac{N}{2}}\right| \psi>}{<\psi\left|T^{N}\right| \psi>}
$$

where $\delta\left(|\sigma>-| \uparrow \ldots \uparrow>_{b}\right)$ fixes the spins in the basis $b$ in the up direction and $\mid \psi>$ is the state at infinity and can be in principle any state not orthogonal to the ground state. The denominator of the above formula is just the total partition of the corresponding two dimensional classical system on the infinite cylinder. The numerator is dependent on the basis that one chooses and in the most simple case of the basis which corresponds to classical variables in the corresponding classical system, for example the $\sigma^{x}$ basis in the transverse field Ising model, it is just a partition function of a two dimensional classical system with fixed boundary conditions on a slit. We will later show that if one chooses the $\sigma^{z}$ basis in the Ising model this will lead us to free boundary conditions on the slit. If the boundary condition imposed by the chosen basis flows to some sort of boundary conformal field theory (BCFT) then one can calculate the logarithmic emptiness formation probability using BCFT techniques. Consider $p_{D}$ as the probability of formation then we have the following formula 
for logarithmic emptiness formation probability $\Pi_{D}$ of a subsystem with size $s[9$

$$
\Pi_{D}:=-\ln p_{D}=\alpha s+\frac{c}{8} \ln s+\ldots,
$$

where $\alpha$ is a non-universal constant, $c$ is the central charge of the system and the dots are the subleading terms. In 10 it was shown that the method not only works for the emptiness formation probability but also for much more general formation probabilities. This is just because there are many different configurations that flow to BCFT in the scaling limit. It is quite obvious that the method can be generalized to cases in which the domain $D$ is not a connected domain. The next simple case is the formation probability of two-disjoint intervals $A$ and $B$. In this case, consider the configurations $\mathcal{C}_{A}$ and $\mathcal{C}_{B}$ in the intervals $A$ and $B$ respectively. First of all in principle the configurations $\mathcal{C}_{A}$ and $\mathcal{C}_{B}$ not only can be different, they also can be considered in different bases. Following [9] and the argument given for one interval one can simply write

$$
\mathcal{R}(A, B):=-\ln \frac{p\left(\mathcal{C}_{A}, \mathcal{C}_{B}\right)}{p\left(\mathcal{C}_{A}\right) p\left(\mathcal{C}_{B}\right)}=-\ln \frac{Z^{A B} Z}{Z^{A} Z^{B}},
$$

where $\mathcal{R}$ is defined based on the first equality. $p\left(\mathcal{C}_{A}, \mathcal{C}_{B}\right)$ is the corresponding probability of two disjoint intervals $A$ and $B$ and $p\left(\mathcal{C}_{A}\right), p\left(\mathcal{C}_{B}\right)$ are the corresponding probabilities of the intervals $A$ and $B . Z^{A}, Z^{B}$ and $Z^{A B}$ are the partition functions of the whole $2 d$ classical system with slits on $A, B$ and $A B$ respectively and $Z$ is the total partition function. Note that the normalization is in a way that for $A$ and $B$ very far from each other $\mathcal{R}(A, B)$ goes to zero. It is worth mentioning that depending on the configurations $\mathcal{C}_{A(B)}$ and the bases the partition functions $Z^{A(B)}$ can be different because different configurations in different bases can induce different boundary conditions on the slits. However, as we will show in the example of the Ising model there are a lot of configurations that in the scaling limit flow to the same boundary conditions which means that the leading universal term of $\mathcal{R}(A, B)$ is the same for all of these configurations.

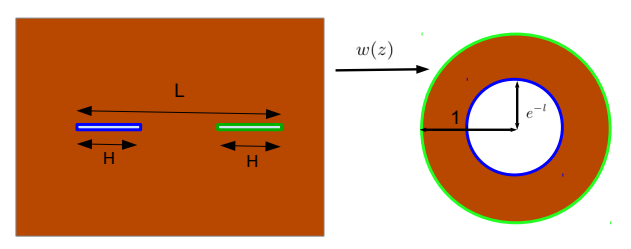

Fig. 1: The conformal map $w(z)$ sends the plane with two slits to the annulus.

It is not difficult to see that the quantity $\mathcal{R}(A, B)$, by definition [12, is just the Casimir free energy of two needles in a $2 d$ system. The Casimir free energy for arbitrary shapes in two dimensional critical systems is calculated using conformal field theory techniques in 12,13 . The idea is based on mapping the $2 d$ system with two removed domains to the annulus and then using the partition function of CFT on the annulus. Following this procedure the 
Casimir free energy can be written as 12,13 :

$$
\mathcal{R}(A, B)=\mathcal{F}_{\text {ann }}+\mathcal{F}_{\text {geo }},
$$

where $\mathcal{F}_{a n n}$ is the free energy of CFT on the annulus which is known for most of CFT's 18 and $\mathcal{F}_{\text {geo }}$ can be calculated using 13

$$
\frac{\delta \mathcal{F}_{\text {geo }}}{\delta H}=\frac{i c}{6 \pi} \oint_{\partial S_{2}}\{w, z\} d z
$$

where $S_{2}$ is a contour surrounding one of the domains $A(B)$ and $\{w, z\}=\frac{w^{\prime \prime \prime}}{w^{\prime}}-\frac{3}{2}\left(\frac{w^{\prime \prime}}{w^{\prime}}\right)^{2}$ is the Schwarzian derivative. Note that in this work we keep $L$ fixed and vary $H$. To calculate the logarithmic formation probability of two disjoint intervals one just needs to apply the above equations to the problem of two slits. The conformal map from the plane with two cuts on a line with lengths $H$ and the distance $L-2 H$ to the annulus with the inner and outer radiuses $r=e^{-l}$ and $r=1$, see Figure 1 , is given by ${ }^{1}$

$$
\begin{aligned}
w(z) & =e^{-\frac{l}{2}} e^{l \frac{\mathrm{sn}^{-1}\left(\frac{2 z}{d}, k^{2}\right)}{2 \mathcal{K}\left(k^{2}\right)}}, \\
l & =2 \pi \frac{\mathcal{K}\left(k^{2}\right)}{\mathcal{K}\left(1-k^{2}\right)},
\end{aligned}
$$

where $\mathcal{K}$ and $\mathrm{sn}^{-1}$ are the elliptic and inverse Jacobi functions ${ }^{2}$ respectively and $k=\frac{d}{L}$ and $d=L-2 H$. After performing the integration, we will finally have

$$
\mathcal{F}_{\text {geo }}=\frac{c}{12 L} \int_{a}^{H} d h \frac{L^{2} \pi^{2}-2\left(h^{2}-4 h L+2 L^{2}\right) \mathcal{K}^{2}\left(\frac{4 h(L-h)}{L^{2}}\right)}{\left(2 h^{2}-3 h L+L^{2}\right) \mathcal{K}^{2}\left(\frac{4 h(L-h)}{L^{2}}\right)}
$$

where $a$ plays the role of lattice spacing.

The annulus contribution part for a generic CFT with boundary conditions $b$ and $d$ on the two boundaries has the following form 18

$$
\mathcal{F}_{a n n}=-\ln Z_{b d}(\tilde{q})=c \frac{l}{12}-\ln \sum_{h}\left|b_{h}\right|^{2} \chi_{h}\left(\tilde{q}^{2}\right),
$$

where $\tilde{q}=e^{-l}$ and $Z_{b d}(\tilde{q})$ is the partition function on the annulus. $\chi_{h}\left(\tilde{q}^{2}\right)$ is the character of the conformal operator with conformal weight $h$ and $b_{h}$ is a number. It is worth mentioning that for large distances we will have $\mathcal{F}_{a n n} \sim \frac{1}{L^{2 x} b d}$, where $x_{b d}$ is the smallest eigenvalue of $L_{0}+\bar{L}_{0}$ that couples the boundary conditions $b$ and $d\left[13 . L_{0}\right.$ and $\bar{L}_{0}$ are the Virasoro generators. For the Ising model with free-free $(\mathrm{FF})$ boundary conditions one can write the above formula explicitly as follows:

$$
\mathcal{F}_{\text {ann }}^{F F}=\frac{l}{24}-\frac{1}{2} \log \frac{\Theta\left(3, e^{-l}\right)}{\eta\left(\frac{i l}{\pi}\right)},
$$

where $\Theta$ and $\eta$ are the Jacobi and Dedekind functions respectively. For the $X X$-chain the free energy on the annulus for the Dirichlet-Dirichlet (DD) boundary condition is 19

$$
\mathcal{F}_{\text {ann }}^{D D}=\frac{l}{12}-\log \frac{\sum_{n \in Z} e^{-n^{2} l}}{\eta\left(\frac{i l}{\pi}\right)} .
$$

\footnotetext{
${ }^{1}$ The extension of this result to non-equal slits is straightforward.

${ }^{2}$ Note that in all of the formulas we adopt the Mathematica convention for all the elliptic functions.
} 
We now check the above relations numerically using the method proposed in [10 for the critical XY chain. The Hamiltonian of XY chain is given by

$$
H=-\sum_{j=1}^{L}\left[\left(\frac{1+\gamma}{2}\right) \sigma_{j}^{x} \sigma_{j+1}^{x}+\left(\frac{1-\gamma}{2}\right) \sigma_{j}^{y} \sigma_{j+1}^{y}+h \sigma_{j}^{z}\right]
$$

After the Jordan-Wigner transformation it can be written as

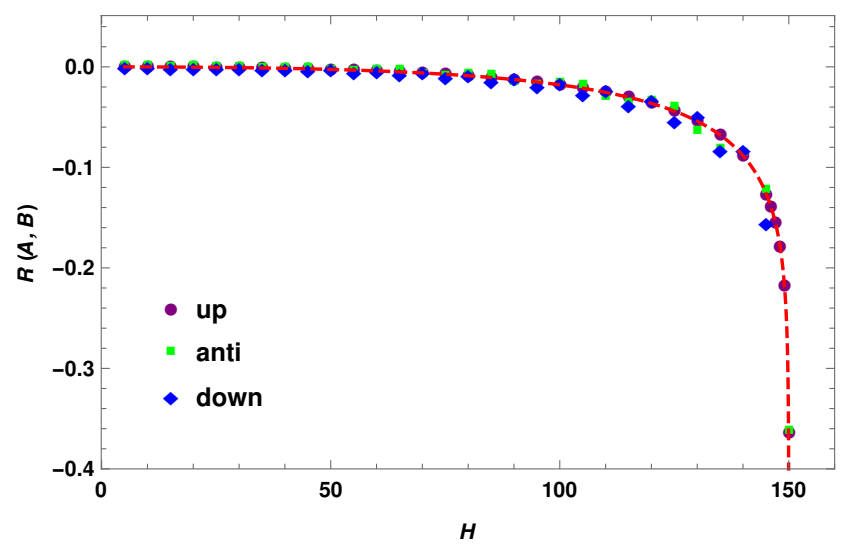

Fig. 2: The quantity $\mathcal{R}$ for two disjoint intervals for configurations with, all spins up, all spins down and antiferromagnetic pattern. The dashed line is the analytical result. The parameter $L=300$ is fixed in our calculations.

$$
H=-\sum_{j=1}^{L}\left[\left(c_{j}^{\dagger} c_{j+1}+\gamma c_{j}^{\dagger} c_{j+1}^{\dagger}+h . c .\right)+h\left(2 c_{j}^{\dagger} c_{j}-1\right)\right]
$$

where for $\gamma=h=1$ we have the critical Ising model and for $\gamma=h=0$ we have the critical XX chain. Note that in the language of free fermions a spin up (down) in the $\sigma^{z}$ basis means the lack (presence) of a fermion in that particular site. Using the coherent states it was shown in $[10$ that the probability of configuration $\mathcal{C}$ for an arbitrary region can be calculated using the following formula

$$
P(\mathcal{C})=\operatorname{det}\left[\frac{1}{2}(1-G)\right] M_{J}^{\mathcal{C}},
$$

where the block Green matrix is defined as $G_{i j}=\operatorname{tr}\left[\rho_{D} b_{i} a_{j}\right]$ with $a_{i}=c_{i}^{\dagger}+c_{i}$ and $b_{i}=c_{i}^{\dagger}-c_{i}$. $M_{J}^{\mathcal{C}}$ is the minor of the matrix $J=(G+1)(G-1)^{-1}$ corresponding to the configuration $\mathcal{C}$. Different configurations correspond to different diagonal minors of the matrix $J$, for example, for the configuration of all spins up the emptiness formation probability will be just $\operatorname{det}\left[\frac{1}{2}(1-G)\right]$ which we used the minor with the rank $k=0$. The method to extract the probability of other configurations are explained in [10]. The algorithm of the calculation is as follows: for any spin up in the configuration one just needs to remove the corresponding row and column in the matrix $J$ and then calculate the determinant. We applied this procedure to calculate the formation probability of two disjoint intervals in the critical Ising model with $G_{i j}=-\frac{1}{\pi(i-j+1 / 2)}$. It is expected that the configurations of all spins up (down) and alternating antiferromagnetic configuration flow to free-free conformal boundary conditions in the scaling limit. In the case of all spins up the argument for one interval goes as follows: consider an interval with all spins up in the $\sigma^{z}$ basis. To make contact with the classical Ising model we need to write the state with all the spins up in the $\sigma^{x}$ basis as 
follows:

$$
\left|\uparrow \ldots \uparrow>_{z}=\frac{1}{2^{s / 2}}\left(\left|\rightarrow>_{x}+\right| \rightarrow>_{x}\right) \ldots\left(\left|\rightarrow>_{x}+\right| \rightarrow_{x}\right)=\frac{1}{2^{s / 2}} \sum_{\left\{\sigma_{j}^{x}\right\}}\right| \sigma_{1}^{x} \ldots \sigma_{s}^{x}>=\mid \text { free }>
$$

The above formula demonstrate that all the spins up configuration in the Euclidean version is just a free boundary condition. Generalization of the above argument to arbitrary intervals is straightforward. Although the above calculation is exact for the case of all spins up the same argument does not go smoothly for all spins down and antiferromagnetic configurations. By numerical calculations it is shown in 10 that these configurations also flow to conformal boundary conditions. Here we show that numerical calculations are consistent with the free boundary conditions for all the considered configurations in the $\sigma^{z}$ basis. Our numerical results depicted in the Figure 2 show that the $\mathcal{R}(A, B)$ is given by (4) with $\mathcal{F}_{\text {geo }}$ and $\mathcal{F}_{\text {ann }}$ given by $(8)$ and $(10)$. We also realized that the contribution coming from $\mathcal{F}_{\text {geo }}$ (which we evaluated numerically) is extremely small and one can get compatible results just by considering the $\mathcal{F}_{\text {ann }}$. However, one should notice that this term can be significant for needles in very small distances. It is worth mentioning that the two configurations - all the spins down and the antiferromagnetic one - show small oscillating behaviour which is a consequence of the parity effect [10]. In the case of XX chain, we considered just the

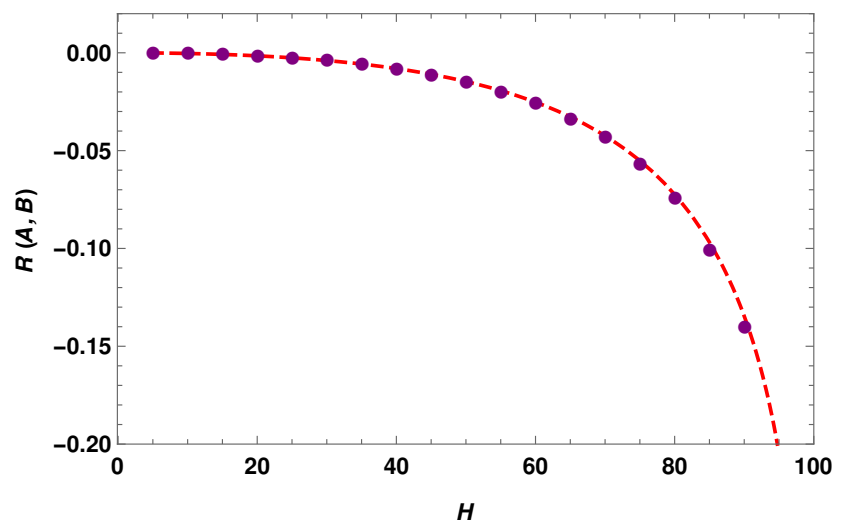

Fig. 3: The quantity $\mathcal{R}$ for two disjoint intervals in the XX chain with respect to $H$ for antiferromagnetic configuration. The dots are the data coming from numerical calculations and the solid line is the analytical result. The parameter $L=200$ is fixed in our calculations.

alternating configuration which is expected to flow to the DD boundary condition in the Luttinger liquid language [19]. Note that the configuration with all the spins up does not flow to BCFT and so it is not going to follow the above formulas. The reason behind this fact is that XX chain has a $U(1)$ symmetry which means that the number of fermions is fixed. To have a configuration with all spins up one needs to have a string of empty sites which is not compatible with the half filling picture of XX chain, for more precise argument based on arctic circle phenomena see [9]. Note that the antiferromagnetic configuration is perfectly consistent with the $U(1)$ symmetry of the system. We repeated the same procedure as above with $G_{i j}=\left(1-\delta_{i j}\right) \frac{2}{\pi(i-j)} \sin \frac{\pi(i-j)}{2}$ for antiferromagnetic configuration. The results depicted in Figure 3 show that the $\mathcal{R}(A, B)$ is given by (4) with $\mathcal{F}_{\text {geo }}$ and $\mathcal{F}_{\text {ann }}$ given by (8) and (11).

We finally comment on the Rényi mutual information of two disjoint intervals. The Rényi entropy of the region $\mathcal{X}$ is defined as

$$
S h_{n}(\mathcal{X})=\frac{1}{1-n} \ln \sum_{I} p_{I}^{n}
$$


where $p_{I}$ is the probability of having a particular configuration in the system and the sum is over all the possibilities. Then the naive definition of the Rényi mutual information between the regions $A$ and $B$ is as follow ${ }^{3}$.

$$
I_{n}(A, B)=S h_{n}(A)+S h_{n}(B)-S h_{n}(A \cup B) .
$$

As it is argued in 9] when $n \rightarrow \infty$ only the biggest contribution survives and so one can drop the sum in the definition of the Rényi information. The configuration with the highest probability in the case of the transverse-field Ising model is the one with all the spins up. However, For XX-chain the most important probability is the antiferromagnetic configuration. As we already discussed both cases are related to BCFT and their formation probabilities are given by the formulas that we discussed above. After a bit of algebra, for $n \rightarrow \infty$ we have

$$
I_{n}(A, B)=\frac{n}{1-n} \mathcal{R}(A, B) .
$$

Although the above result is derived for $n \rightarrow \infty$, its validity, as in the case of one interval [3] 21], might extend up to finite $n>1$.

Conclusions: The simple connection between the formation probabilities and the Casimir effect that we presented here enabled us to find an exact solution for the formation probabilities of two disjoint intervals in generic one dimensional quantum critical systems. The quantity is of great interest because it has a lot of information regarding the universality class of the system. The connection that we presented also gives a very efficient method to numerically calculate the Casimir energy between two needles in a two dimensional medium. We also commented on the connection between Rényi mutual information and the Casimir effect. It will be very interesting to generalize the results to different models with different boundary conditions. For example studying the Neumann-Neumann boundary condition in the XX chain [19] is of great interest. In addition, here we just commented on the formation probabilities of the XY-chain in the $\sigma^{z}$ basis, one needs to also study other bases such as $\sigma^{x}$ basis which in the case of the Ising model is just the fixed-fixed boundary condition [12]. Numerical study of other bases is a big challenge because one cannot in general use the free fermion representation to calculate them. Finally it will be also interesting to extend some of these results to higher dimensions.

I thank M F Maghrebi, K Najafi and T Oliveira for discussions. This work was supported in part by CNPq.

\section{REFERENCES}

[1] Blöte H. W. J. and Cardy J. L. and Nightingale M. P., Phys. Rev. Lett. , 56 (1986) 742; AfFleck I., Phys. Rev. Lett., 56 (1986) 746

[2] Holzhey C. and Larsen F., Wilczek F., Nuclear Physics B, 424 (1994) 443; Calabrese P. and Cardy J., J. Stat. Mech., 2004 ( P06002)

[3] Alcaraz F. C. and Rajabpour M. A.,, Phys. Rev. Lett., 111 (2013) 017201; Alcaraz F. C. and Rajabpour M. A., Phys. Rev. B, 90 (2014) 075132

[4] Korepin V. E. and Izergin A. G. and Essler F. H. L. and Uglov D. B., Phys. Lett. A, 190 (1994) 182; Essler F. H. L. and Frahm H. and Izergin A. G. and Korepin V. E., Comm. Math. Phys., 174 (1995) 191

[5] Kitanine N. and Maillet J-M. and Slavnov N. and Terras V., J. Phys. A: Math. Gen, 35 (2002) L385; Kitanine N. and Maillet J-M. and Slavnov N. and Terras V., J. Phys. A: Math. Gen., 35 (2002) L753; Abanov A. G. and . Korepin V. E, Nucl. Phys. B., 647 (2002) 565; Korepin V. E. and Lukyanov S., Nishiyama Y. and Shiroishi M., Phys. Lett.

\footnotetext{
${ }^{3}$ For the distinction between different mutual Rényi informations see 20
} 
A , 312 (2003) 21; Kozlowski K. K.,J. Stat. Mech., 2008 (P02006) ; Cantini L., J. Phys. A: Math. Theor., 45 (2012) 135207

[6] Abanov A.G. and Korepin V. E., Nucl. Phys. B, 647 (2002) 565

[7] Shiroishi M. and Takahashi M. and Nishiyama Y., J. Phys. Soc. Jpn., 70 (2001) 3535

[8] Abanov A. G. and Franchini F., Phys. Lett. A, 316 (2003) 342; Abanov A. G. and Franchini F.,J. Phys. A, 38 (2005) 5069

[9] Stéphan J-M , J. Stat. Mech., 2014 ( P05010)

[10] NAJAfi K. and Rajabpour M. A., arXiv:1511.06401

[11] Calabrese P., Cardy J., Tonni E. , J. Stat. Mech, 2011 (1101:P01021) ; Headrick M., Phys. Rev. D, 82 (2010) 126010; Calabrese P., Cardy J., Tonni E., Phys. Rev. Lett. , 109 (2012) 130502; M. A. Rajabpour, F. Gliozzi, J. Stat. Mech., 2012 (P02016 ) ; Coser A, Tonni E, Calabrese P.,[arXiv:1508.00811];

[12] Machta B. B. and Veatch S. L. and Sethna J. P., Phys. Rev. Lett., 109 (2012) 138101

[13] Bimonte G. and Emig T. and Kardar M., EPL, 104 (2013) 21001

[14] Vasilyev O. A. and Eisenriegler E. and Dietrich S., Phys. Rev. E, 88 (2013) 012137

[15] Yolcu C. and Rothstein I. Z. and Deserno M., Phys. Rev. E, 85 (2012) 011140; Bimonte G. and Emig T. and Kardar M., Physics Letters B, 743 (2015) 138

[16] Maghrebi M. F. and Reid H., Phys. Rev. Lett., 114 (2015) 151602; Maghrebi M. F., $\operatorname{arXiv:1510.00018}$

[17] Gambassi A. and Silva A., arXiv:1106.2671; Gambassi A. and Silva A., Phys. Rev. Lett., 109 (2012) 250602; Sotiriadis S. and Gambassi A. and Silva A., Phys. Rev. E, 87 (2013) 052129

[18] Cardy J. , arXiv:hep-th/0411189

[19] Bilstein U., J. Phys. A: Math. Gen., 33 (2000) 4437

[20] Alcaraz F. C. and Rajabpour M. A., Phys. Rev. B, 91 (2015) 155122

[21] StÉphan J-M, Phys. Rev. B, 90 (2014) 045424 